\title{
JacLy: a Jacobian-based method for the inference of metabolic interactions from the covariance of steady-state metabolome data
}

\author{
Mohammad Jafar Khatibipour ${ }^{1,2}$, Furkan Kurtoğlu ${ }^{1}$, Tunahan Çakır ${ }^{\text {Corresp. } 1}$ \\ ${ }^{1}$ Computational Systems Biology Group, Department of Bioengineering, Gebze Technical University, Gebze, Kocaeli, Turkey \\ 2 Department of Chemical Engineering, Gebze Technical University, Gebze, Kocaeli, Turkey \\ Corresponding Author: Tunahan Çakır \\ Email address: tcakir@gtu.edu.tr
}

Reverse engineering metabolome data to infer metabolic interactions is a challenging research topic. Here we introduce JacLy, a Jacobian-based method to infer metabolic interactions of small networks ( $<20$ metabolites) from the covariance of steady-state metabolome data. The approach was applied to two different in silico small-scale metabolome datasets. The power of JacLy lies on the use of steady-state metabolome data to predict the Jacobian matrix of the system, which is a source of information on structure and dynamic characteristics of the system. Besides its advantage of inferring directed interactions, its superiority over correlation-based network inference was especially clear in terms of the required number of replicates and the effect of the use of priori knowledge in the inference. Additionally, we showed the use of standard deviation of the replicate data as a suitable approximation for the magnitudes of metabolite fluctuations inherent in the system. 
1

2

3

4

5

6

7

8

9

10

11

12

17 * Corresponding author

18

19

20 TURKEY

Corresponding author
JacLy: A Jacobian-based Method for the Inference of Metabolic Interactions from the Covariance of Steady-State Metabolome Data

Mohammad Jafar Khatibipour ${ }^{1,2}$, Furkan Kurtoğlu' ${ }^{1}$, Tunahan ÇAKIR ${ }^{1, *}$

${ }^{1}$ Computational Systems Biology Group, Department of Bioengineering, Gebze Technical University, 41400, Gebze, Kocaeli, TURKEY

${ }^{2}$ Department of Chemical Engineering, Gebze Technical University, 41400, Gebze, Kocaeli,

Email address: tcakir@gtu.edu.tr 


\section{Abstract}

Reverse engineering metabolome data to infer metabolic interactions is a challenging research topic. Here we introduce JacLy, a Jacobian-based method to infer metabolic interactions of small networks $(<20$ metabolites) from the covariance of steady-state metabolome data. The approach was applied to two different in silico small-scale metabolome datasets. The power of JacLy lies on the use of steady-state metabolome data to predict the Jacobian matrix of the system, which is a source of information on structure and dynamic characteristics of the system. Besides its advantage of inferring directed interactions, its superiority over correlation-based network inference was especially clear in terms of the required number of replicates and the effect of the use of priori knowledge in the inference. Additionally, we showed the use of standard deviation of the replicate data as a suitable approximation for the magnitudes of metabolite fluctuations inherent in the system.

\section{Introduction}

Inference of cellular interactions by processing biomolecular data is a widely-used approach to investigate functional properties of cellular systems. Perturbations due to genetic/environmental alterations and diseases lead to changes in functionality due to change in cellular network structure, and network inference using the biomolecular data of the perturbation states uncovers the changes in network structure. When applied to the data of metabolite levels, the approach infers metabolic interaction (Srividhya et al., 2007; Çakır et al., 2009; Hendriks et al., 2011; Çakır \& Khatibipour, 2014). The general trend is to use dynamic data to infer directed metabolic networks, and steady-state data to infer undirected networks. On the other hand, there are approaches that infer directed metabolic interactions from steady-state metabolome data by utilizing inherent intrinsic variability in such data (Steuer et al., 2003; Öksüz, Sadıkoğlu \& Çakır, 2013).

While the principles of conservation of mass and conservation of energy set the boundaries for deterministic behavior of the metabolic network, the inherent randomness in this network, as it exists in other biological networks, leads to small variability in the steady-states of the system at equivalent macroscopic conditions (Wu et al., 2005; Kresnowati et al., 2006). From a microscopic point of view, the inherent randomness is believed to be the result of existence of 
52 discrete particles in the system, and molecular fluctuations are inherent in the mechanism by 53 which the system evolves (Kampen, 1992). Continuous change in micro-environment as well as multilevel complex regulatory mechanisms in the metabolic network are also the causes of observed variability in the steady-states of the system (Steuer et al., 2003). Although this intrinsic randomness introduces a great obstacle and difficulty in modeling and simulation of metabolic networks, at the same time it provides an opportunity to infer and estimate the active metabolic network at a specific condition/context just by reverse engineering the corresponding replicates of metabolome data. Considering the fact that information on interactions between metabolites and hence the structure of the active metabolic network is implicit in these data, the main questions are (i) how much information on the structure of the network is hidden in the data, and (ii) how we can extract as much as possible of that information.

One common approach that utilizes inherent variability in steady-state data is correlation based inference methods, especially the Gaussian Graphical Model (GGM) approach (Çakır et al., 2009; Montastier et al., 2015; Wang et al., 2016). Correlation based approaches are capable of detecting strong interactions in the metabolic network to some extent. However, they infer interactions only in undirected manner, and they have limited power in the detection of weak interactions (Çakır et al., 2009). A directed network inference approach from steady-state metabolome data is also available in the literature (Steuer et al., 2003; Öksüz, Sadıkoğlu \& Çakır, 2013; Çakır \& Khatibipour, 2014). The approach is based on the prediction of interaction strengths from the covariance of the data. The network structure information stored in the inherent variability of the data is reflected on the covariance of the data, and later used in the prediction of interaction strengths in the form of a Jacobian matrix. The Jacobian matrix of a cellular interaction system contains a significantly high amount of valuable information both on the structure and dynamic characteristics of the system. Numbers in this matrix easily provide us with detailed information on the underlying interactions in the network, such as direction of interactions, nature of interactions (positive or negative effects), and strengths of interactions (Steuer et al., 2003; Öksüz, Sadıkoğlu \& Çakır, 2013). The Lyapunov equation provides a link between the Jacobian matrix of a cellular system and the covariance matrix of the replicates of steady-state data. This equation is the result of a Langevin type approach for description of stochastic processes at macroscopic level (Scott, 2013). The Lyapunov equation was also used 
83 previously to infer differential changes in a Jacobian matrix rather than the inference of the network structure itself (Sun \& Weckwerth, 2012; Kügler \& Yang, 2014). In another work (Sun, Länger \& Weckwerth, 2015), a comparison of several least square and regularization methods in solving the Lyapunov equation for the Jacobian matrix is provided. However, in that work, the structure of the Jacobian (zero and non-zero elements) is specified a priori by the stoichiometric matrix of the metabolic network. Therefore, the problem is reduced to the estimation of magnitudes for non-zero elements in the Jacobian matrix, which might not be considered as a network inference.

In our previous work, we focused on a theoretical analysis on the applicability of Jacobian-based inference of directed metabolic interactions from steady-state data (Öksüz, Sadıkoğlu \& Çakır, 2013). In silico covariance data were used to predict a Jacobian matrix with an optimization framework based on dual objective function. The objective function was simultaneous maximization of the sparse structure, as observed in cellular networks (Yeung, Tegnér \& Collins, 2002; Tegnér \& Björkegren, 2007; Bordbar et al., 2014; Shao et al., 2015; Mangan et al., 2016), and minimization of the residual norm of the Lyapunov equation (see methods). The theoretical analyses proved the potential usefulness of the approach. Here, we improve the algorithm used in that work considerably in terms of speed and optimality, and apply it successfully to in silico metabolome data.

\section{Methods}

\section{Problem Definition}

Provided that replicates of metabolome data are available for an organism in a specific condition, and considering the fact that information on interactions between metabolites and hence the structure of the metabolic network is implicit in these data, the problem is to extract from the data as much knowledge as possible to infer the active metabolic network in that condition. A metabolic reaction network can be mathematically represented by writing mole-balance equations around its metabolites. This leads to a system of differential equations that can be summarized as in the following equation, where $\mathrm{C}$ is a vector of metabolite concentrations:

$$
\frac{d C}{d t}=f(C)
$$


114 For a system around steady-state, a linear approximation can be made to express the equations in 115 terms of a Jacobian matrix, J (Steuer et al., 2003; Jamshidi \& Palsson, 2008):

116

117

118

$$
\frac{d X}{d t} \approx J X
$$

with $X=C-C_{S}$, and $C$ shows concentrations fluctuating around steady-state values, $C_{S}$. Equation (2) can further be expressed as a Langevin-type equation to explicitly account for small fluctuations (Steuer et al., 2003):

$$
\begin{gathered}
\frac{d X_{i}}{d t}=\sum_{j} J_{i j} X_{j}+\sqrt{2 D_{i}} \delta_{i}(t) \\
J_{i j}=\frac{\partial\left(\frac{d C_{i}}{d t}\right)}{\partial C_{j}}
\end{gathered}
$$

Where $D_{i}$ shows the extent of fluctuation and $\delta_{i}$ is a random number from unit normal distribution. As demonstrated in the literature (Kampen, 1992), equation (3) can be written as follows at steady-state:

$$
J C+C J^{T}=-2 D
$$

Equation (5) is known as the Lyapunov equation, and it provides a link between the Jacobian matrix of the network and the covariance matrix of the replicate metabolome data (Steuer et al., 2003; Öksüz, Sadıkoğlu \& Çakır, 2013).

The fluctuation matrix (D) accounts for the inherent randomness in the system. The diagonal elements of D reflect the magnitude of fluctuations observed on each metabolite, and the nondiagonal elements can be assumed as zero (Steuer et al., 2003). The equation is determined in terms of calculating the covariance matrix (C) while the Jacobian matrix $(\mathrm{J})$ is provided, however, it is underdetermined in the case of calculating $\mathrm{J}$ while $\mathrm{C}$ is available. This is because there are $n(n+1) / 2$ independent entries in $C$ for an $n$-metabolite system due to the symmetric nature of the covariance matrix, whereas the Jacobian matrix has $\mathrm{n}^{2}$ independent entries. This equation can be rearranged to a standard linear system of equations (Öksüz, Sadıkoğlu \& Çakır, 2013) and be represented as follows:

$$
A j+2 d=0
$$


144 In this equation, $\mathrm{A}$ is a square matrix of size $n^{2} \times n^{2}$ derived from the covariance matrix, $\mathrm{j}$ is the 145 vectorized form of the Jacobian matrix with size $n^{2} \times 1$, and $\mathrm{d}$ is the vectorized form of 146 fluctuation matrix with size $n^{2} \times 1$, where $\mathrm{n}$ is the number of metabolites. Öksüz et al. used 147 equation (6) to solve for Jacobian matrix in an optimization platform using Genetic Algorithm 148 (Öksüz, Sadıkoğlu \& Çakır, 2013). Beside the minimization of the residual of Lyapunov 149 equation, they used sparsity as a rational objective function to select Jacobians from the solution 150 space that have a high number of zeros and satisfy equation (6) as well. The multi-objective 151 function that simultaneously maximizes the number of zeros (sparsity) in the Jacobian matrix to 152 153 be determined and minimizes the residual of equation (6) can be represented as follows:

155

The first term in the equation counts for the number of zeros in the Jacobian matrix that needs to be maximized, the second term counts for the residual of Lyapunov equation that needs to be minimized, and in total the objective function $\mathrm{f}$ is to be maximized. Lambda $(\lambda)$ is a scaling factor discussed in detail in a section below. In order to balance between the two goals in the objective function, and also to refrain the solution from going to a very high number of zeros, the scaling factor was introduced in the objective function.

Using the exact covariance and predefined fluctuation vector as inputs to the algorithm, Öksüz et al. (Öksüz, Sadıkoğlu \& Çakır, 2013) validated the theoretical applicability of this approach. Here, we used the same objective function, but after careful examination of the problem we came up with an extensively modified algorithm that is highly robust and could be applied to the replicates of in-silico metabolome data generated by simulating stochastic dynamic models of metabolism using stochastic differential equation (SDE) solvers. The MATLAB function file of the SDE model that was simulated to generate SDE data for yeast is available in Data S1. equipped with a $3.2 \mathrm{GHz}$ CPU and 4GB RAM. SDE simulations were performed using the SDE toolbox that is freely available as an external MATLAB toolbox (Picchini, 2007). Genetic 
174 algorithm (GA) was implemented using the ga function in MATLAB's global optimization 175 toolbox. A built-in parallelized version of ga was used with the help of MATLAB's parallel 176 computing toolbox. Custom MATLAB functions were written for creation, crossover and 177 mutation fields of GA. Maximum number of generations was set to 800 and a mutation rate of $1785 \%$ was selected after careful examination of GA's behavior. The MATLAB codes for JacLy is 179 available in Data S2.

180

181

182

183

184

185

186

187

188

189

190

191

192

193

194

195

196

197

198

199

200

201

202

203

204

\section{Optimization Pipeline}

Genetic Algorithm (GA) was used to solve equation (6) for $\mathrm{j}$ while $\mathrm{A}$ and $\mathrm{d}$ are settled. At each generation of GA, bit string vectors are generated for $\mathrm{j}$ as individuals. With a candidate bit string for the structure of the Jacobian vector (zero and nonzero elements in j), equation (6) can be reduced to a lower dimensional system of equations by removing the zeros in the $\mathrm{j}$ and also removing the corresponding columns in A.

$$
A_{r} j_{r}+2 d=0
$$

Since the Jacobian vector is sparse in structure $(r<<n)$, this leads to considerable reduction in the number of unknowns to be determined, and increases the speed of the inference algorithm compared to the original algorithm in (Öksüz, Sadıkoğlu \& Çakır, 2013). In equation (8), $j_{r}$ is the reduced form of Jacobian vector, obtained by removing those elements corresponding to zeros in the suggested individual, and $A_{r}$ is formed by removing the corresponding columns in A. Equation (8) can be easily treated and solved as a line fitting problem, in which the elements of $j_{r}$ are factors of the line equation and are estimated to make the best fit to the data. Minimizing the Euclidean norm of this fitting is one of the terms in the optimization objective function defined in Equation (7). The other objective is to maximize the number of zeros in the Jacobian matrix, considering the fact that metabolic networks are sparse networks (discussed in a section below).

\section{Constraining the Solution Space by Generating Sparse Individuals}

As the number of metabolites and hence size of the network increases, the solution space expands exponentially and the probability of finding the true candidate for Jacobian vector 
205 through a stochastic algorithm decreases significantly. Moreover, the computational time and 206 effort increases dramatically with the size of the network (Hendrickx et al., 2011). In these 207 situations, it is very important to constrain the solution space as much as possible if we are going 208 to solve the problem (equation 8) in a manageable time. One way to constrain the solution space 209 meaningfully is to control the generation and reproduction of candidate individuals in GA such 210 that those individuals with unwanted characteristics are not produced to be tested at all. Since 211 metabolic networks are naturally sparse networks, setting a minimum sparsity parameter for the 212 generated individuals can be used as a controlling parameter. This was another novelty in the 213 algorithm compared to the original algorithm (Öksüz, Sadıkoğlu \& Çakır, 2013).

215 It is known that metabolic networks are highly sparse, meaning that there are much less interactions (edges) in the network compared to the maximum possible number of edges (fully connected network). We calculated the natural sparsity in several known metabolic networks and we could see that all tested networks have a sparsity larger than 0.55 , so a minimum of $50 \%$ was selected as the default value for the minimum sparsity parameter in our algorithm. Just by definition of such a parameter, the solution space to search for non-zero values of Jacobian is greatly reduced. Sparsity parameter was defined as the following:

222

sparsity $\equiv \frac{\text { Total number of possible edges in the network - number of edges in the network }}{\text { Total number of possible edges in the network }}$ $\cong \frac{\text { Number of zeros in the Jacobian }}{\text { Total number of elements in the Jacobian }}$

Based on this definition, a sparsity value of one will mean a network with not even a single edge whereas a value of zero will correspond to a complete digraph. It must be considered that the sparsity calculated from the Jacobian and the one calculated from the biochemical reaction network are not necessarily the same, since the Jacobian also counts for the regulatory interactions which are absent in the biochemical reaction network, but since the number of regulatory interactions is usually insignificant compared to the number of reactions, the two values are very close. 
234 In order to minimize the computational effort and time, we used the minimum sparsity parameter

235 as the control parameter in the creation of the initial population in GA, and then in the production 236 of individuals at subsequent generations. To this goal, custom MATLAB functions were written 237 and used for creation, crossover and mutation fields of GA in MATLAB. This was another 238 novelty over the previous algorithm (Öksüz, Sadıkoğlu \& Çakır, 2013). With this supervised 239 control of individuals, bitstrings with unwanted characteristics had no chance to appear as the candidates for Jacobian vector, and it provided a significant contribution in constraining the solution space. The custom functions for GA were written in such a way that minimum sparsity is intrinsic in the generation of individuals and no time is consumed for control and filtering of the generated bitstrings.

\section{Scanning for the scaling factor}

The objective function (equation 7) consists of two terms, one is the residual of equation (8) to be minimized and the other is the number of zeros in the Jacobian vector to be maximized. In order to balance between these two values and also to prevent the optimization algorithm from converging to the too sparse solutions, a scaling factor $(\lambda)$ is multiplied with the term for the number of zeros in the Jacobian vector. Since this parameter directly affects the magnitude of the objective function, it is important to find a range of lambda that leads to sensible solutions. Selecting very small values for the scaling factor leads to the conditions in which the optimization will not be very sensitive to the number of zeros in the Jacobian vector, and minimization of the residual of equation (8) would be dominant in the objective function. On the other hand, large magnitudes of the scaling factor lead to the solutions with very high number of zeros in the Jacobian vector, with almost no sensitivity to the residual of equation (8). There is always a narrow interval for the scaling factor, in which the optimization problem can find a Jacobian vector with optimum number of zeros that also leads to insignificant residual value for equation (8). This interval for the scaling factor varies from problem to problem (Öksüz, Sadıkoğlu \& Çakır, 2013), depending on several factors among which are the size of the network and the accuracy and number of data replicates from which covariance matrix is calculated. Constant problem-specific $\lambda$ values were used in the previous algorithm (Öksüz, Sadıkoğlu \& Çakır, 2013). In order to circumvent the obstacles due to selection of a proper value for the 
265

266

267

268

269

270

271

272

273

274

275

276

277

278

279

280

281

282

283

284

285

286

287

288

289

290

291

292

293

294

scan a range of values for this parameter in this work in an unsupervised manner. We scanned a range roughly between $0.01-0.10$, with increments of 0.005 . Since we repeated the algorithm 10 times due to the stochastic nature of genetic algorithm, this led to a total of about 200 solutions per network inference problem. In this way, the optimization algorithm works repeatedly for each value of the scaling factor, and the optimum solution would have chance to appear among the candidate solutions. This was another improvement over the previous algorithm.

\section{Fluctuation Vector}

One of the major obstacles in utilizing the Lyapunov equation is introduced by the fluctuation matrix D since it may contain non-observable quantities (Kügler \& Yang, 2014). The fluctuation matrix plays a critical role in this equation, and the computed Jacobian matrices are highly sensitive to the values in the fluctuation matrix. After a reasonable fluctuation matrix is selected, the problem of solving the underdetermined equation to find the Jacobian can be formulated as an optimization problem.

The existence of a non-zero fluctuation vector is both physically and mathematically meaningful. Fluctuation vector represents the intrinsic noise in the molecular interactions that are the source of stochasticity in the replicates of data through which we are going to extract information. A non-zero fluctuation vector also prevents the equation (6) to have null space, that otherwise would be problematic. On the other hand, it is not very clear how to find and how to set the values for the fluctuation vector in equation (6). In a previous work (Öksüz, Sadıkoğlu \& Çakır, 2013), a constant problem non-specific small value of 0.005 was used for all metabolites to mimick small fluctuations around metabolites. Here, we hypothesize that standard deviation of the data replicates would be an acceptable candidate to be selected as the diagonal elements of the vectorized fluctuation matrix. The use of data-specific fluctuation vector elements in this manner rather than using a constant value for all problems is another improvement in this algorithm compared to the original algorithm (Öksüz, Sadıkoğlu \& Çakır, 2013).

Using a community of estimated Jacobians instead of only one elite Jacobian to infer the structure of the network 
295 JacLy scans a range of scaling factors $(\lambda)$ in the objective function (Eq. 3). For each scaling 296 factor, optimization is performed 10 times, leading to hundreds of optimizations. The end result 297 of each optimization is a Jacobian vector that has the maximum objective function value among 298 thousands of other individuals. We call this Jacobian as the best-found Jacobian for each 299 optimization. Among all the best-found Jacobians, one can be selected as the elite Jacobian 300 vector based on both sparsity and residual of equation (8). In all the test studies, we could

301

302

303

304

305

306

307

308

309

310

311

312

313

314

315

316

317

318

319

320

321

322

323

324

325 observe that if, instead of the elite Jacobian, we combine a community of the best-found Jacobians in a bounded area around the elite Jacobian and use that community to infer the structure of final Jacobian, the accuracy of inference significantly increases. To do so, first a bounded area is defined around the elite Jacobian based on the number of zeros and the residual of equation (8). For all of the tests in this study, $-/+5 \%$ of the number of zeros in the elite Jacobian was selected to set the lower and upper vertical boundaries and $-5 \%$ of the residual of equation (8) for the elite Jacobian was selected to set the lower horizontal boundary (see figure 1A). The Jacobian vectors in the bounded area are then binarized by setting their non-zero elements to one. The binarized vectors are then aligned on top of each other to form a binary matrix. Taking average over columns of this matrix leads to a new vector including fractional numbers between 0 and 1 . The structure of the final Jacobian vector is then determined by setting a threshold of 0.5 to decide for zero and non-zero values. Those elements that are smaller than the threshold are set to zero and others to one. A looser threshold of 0.4 increases both TPR and FPR. We observed that selecting 0.4 as the threshold leads to better g-scores in general, however, there is the risk of sparsity being dropped to significantly lower values. So, we advise the use of sparsity check as a caution while selecting a threshold for the combination of best-found Jacobians. Figure 1B provides a schematic of this procedure.

In this study, we are not providing a mathematical proof of how using a community of bestfound Jacobians around the elite Jacobian can improve the inference results. However, as far as we tested with different in-silico datasets and noisy covariances, using such combinatorial approach not only leads to better inference results, but also it stabilizes the final output of the algorithm when applied to the same problem repeatedly. The use of a community of best-found Jacobians is another novelty over our previous work (Öksüz, Sadıkoğlu \& Çakır, 2013), which reported the results based on only the elite Jacobian. 


\section{Quantification of inference performance}

328 While True Positive Rate (TPR) and False Positive Rate (FPR) are two quantities suitable for the 329 evaluation and comparison of network inference results, g-score can be used as a single 330 parameter to quantify performance of any inference method. g-score is calculated by the 331 following equation:

332

333

334

335

336

337

338

339

340

341

342

343

344

345

346

347

348

349

350

351

352

353

354 355

$$
g-\text { score }=\sqrt{T P R \times(1-F P R)}
$$

\section{Results}

\section{Use of in silico Covariance Matrix for metabolic models of $S$. cerevisiae and $E$. coli}

The Lyapunov equation (equation (5), and equation (6) in the rearranged form) is underdetermined in terms of calculating the Jacobian matrix $\mathrm{J}$ given $\mathrm{C}$ and $\mathrm{D}$ as inputs, meaning that there is more than one Jacobian matrix satisfying the Lyapunov equation for each pair of $\mathrm{C}$ and D (see Methods section). To evaluate the applicability and performance of our method (JacLy) in predicting the network structure through the prediction of the Jacobian matrix, first we used two kinetic models that are well known in the literature. The first model covers 13 metabolites of yeast glycolysis (Teusink et al., 2000), and the second model covers 18 metabolites of central carbon metabolism in E.coli (Chassagnole et al., 2002). True Jacobian matrix was calculated for each kinetic model around its corresponding steady-state by using the detailed rate expressions and parameters in the models. Here we followed the strategy in our previous work (Öksüz, Sadıkoğlu \& Çakır, 2013), where we tested our genetic-algorithm-based dual objective formulation in terms of the predictability of the Jacobian matrix by using exact covariance matrices as input rather than deriving covariance matrices, from in silico data for example. Our goal in this section is solely to demonstrate the improvements in the current version of our algorithm compared to the previously published algorithm (Öksüz, Sadıkoğlu \& Çakır, 2013). Having the true Jacobian matrix and predefined fluctuation matrices, the exact covariance matrix was calculated from equation (5). We call these covariance matrices as "exact" covariances since they hold true to the Lyapunov equation. Exact covariances and 
356

357

358

359

360

361

362

363

364

365

366

367

368

369

370

371

372

373

374

375

376

377

378

379

380

381

382

383

384

385

386

corresponding fluctuation matrices were then used as inputs to JacLy to evaluate its performance in finding $\mathrm{J}$.

JacLy uses genetic algorithm for optimization, which is a stochastic optimization algorithm. Therefore, it is important to solve for the equation for enough number of times until a constant reproducibility parameter is achieved. For both models, a constant reproducibility is obtained after 20 runs. Out of 20 repetitive runs for each model, 19 and 18 of them could find Jacobian matrices that are in complete agreement (100\% TPR and 0\% FPR) with the true networks of yeast and E.coli models, respectively. These results show a great improvement over the previous work (Öksüz, Sadıkoğlu \& Çakır, 2013), which had a reproducibility parameter of 50\% and 5\% for yeast and E.coli models, respectively. On our desktop computer, each run takes around two minutes for the yeast model and six minutes for E.coli, showing a 10 fold increase in computational speed over the previous work (Öksüz, Sadıkoğlu \& Çakır, 2013). Such significant improvements in reproducibility and computational speed have been achieved solely by modifying the algorithm and corresponding functions (see Methods section). One should note that since JacLy incorporates a $\lambda$ scan with 10 replicate solutions, the whole process of generating 200 solutions for S. cerevisiae took one hour while the timein the case of E.coli was two hours.

We also evaluated the performance of JacLy on noisy covariances. To this goal, we used the exact covariance of the yeast model and followed the same procedure as the previous work (Öksüz, Sadıkoğlu \& Çakır, 2013) to make noisy data. Random numbers were sampled from a normal distribution with a mean of 1 and a standard deviation of 0.005 . This corresponds to a dataset with 50\% noise (Fuente et al., 2004). The random numbers were then symmetrically multiplied with the elements of the exact covariance to generate a noisy covariance matrix. This was repeated to generate ten different noisy covariances and JacLy was applied on each. The average TPR and FPR are 74\% and 5\%, respectively. These numbers were $73 \%$ TPR and 11\% FPR in the previous work (Öksüz, Sadıkoğlu \& Çakır, 2013). These results show a considerable increase in the performance of JacLy compared to its ancestor in terms of the FPR value since exactly the same problem was solved with only improvements in the algorithm based on (i) the use of reduced form of the Lyapunov equation, (ii) the use of sparsity constraint, (iii) scanning 
387 the scaling factor and (iv) the use of a community of candidate Jacobian vectors, as discussed in

388 detail in the Methods section. Additionally, note that a threshold of 0.4 in the combination of 389 Jacobian vectors rather than 0.5 leads to a TPR of $84 \%$ and an FPR of $8 \%$.

390

391 It was reported in the literature that statistical methods such as LASSO and Tikhonov 392 regularization fail to solve equation (6) whenever the condition number of matrix $\mathrm{A}$ is

393

394

395

396

397

398

399

400

401

402

403

404

405

406

407

408

409

410

411

412

413

414

415

416

417 significantly large (Sun, Länger \& Weckwerth, 2015). In order to evaluate the sensitivity of our method to the condition number of $\mathrm{A}$, we used different fluctuation matrices along with the true Jacobians of yeast and E.coli models as inputs to equation (5), and different exact covariances were calculated leading to different A matrices covering a range of condition numbers from $10^{6}$ to $10^{25}$. JacLy was applied to each of those covariance matrices along with their corresponding fluctuation matrices. We could see that the condition number of A doesn't have any influence on the performance of our algorithm. Even for the largest condition numbers, JacLy was able to find the true Jacobian with similar computational time and reproducibility parameters. It should be kept in mind that not being sensitive to the condition number of A in solving equation (6) doesn't mean that the calculated Jacobian matrix is not sensitive to the changes in the fluctuation matrix. Indeed, equation (6) is frequently ill-conditioned as it is also reported in other studies (Sun, Länger \& Weckwerth, 2015). Small changes in the fluctuation matrix D lead to big changes in the calculated Jacobian matrix.

\section{Use of in silico Metabolome Data for metabolic models of $S$. cerevisiae and $E$. coli}

At this stage we applied JacLy to the replicates of in silico metabolome data. Stochastic versions of yeast (Teusink et al., 2000) and E.coli (Chassagnole et al., 2002) models were used to generate 1000 replicates of steady-state metabolome data in silico. In this case we had to come up with a fluctuation matrix to be used as input to the method along with the covariance of metabolome data. As it was mentioned in the Methods section, we hypothesized that standard deviation of data might be a reasonable source to be used for the construction of a fluctuation matrix. In a stochastic dynamic system all or some of the sources of stochasticity are usually unknown. In the Lyapunov equation the fluctuation matrix D is the parameter counting for sources of stochasticity. Since standard deviation is a measure of variation in data, we used it as a reasonable source to construct the fluctuation matrix. Table 1 shows the inference results of 
418 JacLy applied to in-silico data for the yeast and E.coli with a comparison to GGM-based

419 inference results. In GGM analysis a cut-off of 0.01 was used for p-values to decide on the 420 significance of partial Pearson correlation values. The networks predicted by JacLy are directed 421 while those estimated by GGM are undirected.

422

423 It must be considered that solving equation (6) for the Jacobian vector is highly sensitive to the 424 fluctuation vector $\mathrm{d}$, and so it is of critical importance to come up with a fluctuation vector that is 425 most reasonable for data replicates. We thought of normalization as a way that might improve 426 the correspondence between the covariance matrix $\mathrm{C}$ and the fluctuation matrix $\mathrm{D}$ in the 427 Lyapunov equation. Data normalization doesn't have any effect on the results of similarity-based 428 inference methods such as GGM. In-silico metabolome data for the yeast and E. coli were 429 430 431 432 433 434 normalized to between 0 and 1 by dividing each value to the maximum value in the dataset. Normalized data was then used to make both covariance matrix C and fluctuation matrix D. When applied to the normalized data, JacLy showed a significant improvement in inference results for the yeast data (0.95 TPR and 0.13 FPR, with a g-score of 0.91$)$ while it had no effect on the inference results of E.coli data.

Another parameter influential on the result of network inference is the number of replicates in the data. Previous GGM-based analysis for the inference of metabolic interactions using in silico metabolome data for the same networks analyzed here showed a sharp decrease in the quality of the inference after the number of replicates decreased below 200 (Çakır et al., 2009). Here we tested the effect of number of datapoints on the inference results of JacLy. Of 1000 replicates initially generated by stochastic differential equations, 100 randomly chosen replicates were used in the inference of the network for S. cerevisiae. Repeating this 10 times and taking the average, a TPR of 0.73 and an FPR of 0.16 was obtained by using JacLy, corresponding to a g-score of 0.78. On the other hand, GGM-based inference for the same randomly chosen 100 replicates resulted in average TPR and FPR values of 0.42 and 0.03 , respectively, with a g-score of 0.63 . Therefore, an advantage of JacLy over GGM is its considerable robustness in terms of the number of replicate datapoints used in the covariance/correlation calculation. 
448 In the process of inferring a network for a set of metabolites, there are cases when existence (true 449 positive) or non-existence (true negative) of an edge between two metabolites might be available 450 as prior knowledge. Such information can be used as additional input to inference algorithms, 451 resulting in a shrinkage of the solution space and so enhancing the computational speed and 452 performance of the algorithm. We tested the effect of prior knowledge for non-existent edges on 453 the performance of JacLy. To this aim, 7 and 20 zeros of the true Jacobian matrices were 454 selected as priorly known true negatives for yeast and E. coli models, respectively. This 455 corresponds to $5 \%$ and $7 \%$ of the total number of elements in Jacobian matrices for yeast and $E$. 456 coli models. This procedure was repeated 10 times for each model and JacLy was applied to data 457 each time. The average TPR and FPR over 10 repetitions for the yeast model are 0.75 and 0.08 , 458 respectively, leading to a g-score of 0.83 . For E. coli, an average TPR of 0.79 and an average 459 FPR of 0.31 was obtained, leading to a g-score of 0.74 . These results show a significant 460 improvement compared to the corresponding values in Table 1. Based on the results, JacLy 461 performs considerably better when a very small portion of true negatives is introduced as prior knowledge. Specifying true negatives contributed to a better prediction of true positives. The correlation-based GGM approach, on the other hand, is not suitable for the use of prior knowledge.

In addition to the binary structure of estimated Jacobians, which is the main output in inferring the structure of an active metabolic pathway, we also compared the best-found Jacobians in the selected area around the elite Jacobian - before binarization and combination - with the true parameter values in those models. Since JacLy has a stochastic nature, we repeated the optimization on the same SDE data three times, and then we used the Spearman correlation to make the comparisons. The medians of correlations are around 0.45 and 0.25 for yeast and $E$. coli models, respectively while the medians of p-values are less than 0.0001 in all cases (See Fig. $\mathrm{S} 1)$.

We used the kinetic-model-based true Jacobian matrices together with SDE-data-based covariance matrices in theLyapunov equation to calculate D and see its similarity with our approximation for D. We observed that the calculated D contained off-diagonal non-zero 
479 elements as opposed to the approximated D. Some of the elements had the same magnitude as 480 the diagonal elements. On the other hand, our very simple method of estimating D led to quite

481

482

483

484

485

486

487

488

489

490

491

492

493

494

495

496

497

498

499

500

501

502

503

504

505

506

507

508

509

acceptable TPR and FPR values in those case studies, and the standard deviation of data is logically related to the source of natural fluctuation in the system. Therefore, our estimation approach can be used because of its simplicity and applicability. However, research should be performed to develop a more accurate method of estimating D. On the other hand, one should note that SDE simulator algorithms and toolboxes, such as the one used in this study, have stability problems in terms of the generated noise when applied to highly nonlinear systems. This could also be another reason behind the inconsistency between the covariance of SDE data and the true Jacobian matrix, which directly affects calculation of the fluctuation matrix from the Lyapunov equation.

\section{Discussion}

JacLy is a network inference algorithm with specific focus on the inference of small-scale metabolic networks from steady-state data. It has significant advantages over its ancestor (Öksüz, Sadıkoğlu \& Çakır, 2013). Here, we reported algorithmic improvements included in JacLy, which led to significant improvements in the runtime and prediction power. Major improvements were (i) Vectorizing all possible computations, significantly improving the runtime, (ii) the use of the reduced form of the Lyapunov equation by removing the columns corresponding to zero Jacobian vector entries, improving the runtime, (iii) the use of sparsity constraint in genetic algorithm to improve the runtime by eliminating the possibility of generating low-sparsity individuals, (iv) scanning the scaling factor rather than fixing it for each specific problem, making the algorithm more flexible and independent from the effect of chosen parameter value and improving the prediction power, and (v) the use of a community of candidate Jacobian vectors rather than using the elite Jacobian vector in the inference, improving the prediction power of the results. Inferring metabolic pathways via prediction of Jacobian matrices is also useful in estimating dynamic and mechanistic characteristics of the system under investigation.

One issue that is worth mentioning at this stage is the applicability of the approach in terms of the size of the network to be inferred. For example, each run for the E.coli model consumed about twice more computational time compared to that of yeast, while the E.coli model has only 
510 five more metabolites compared to the yeast model - an almost $40 \%$ increase in the number of

511 network nodes. This dramatic increase in computational time with respect to network size 512 whenever the calculation of Jacobian matrix is involved in a network inference method - was 513 observed and explained in previous studies (Hendrickx et al., 2011), and it is indeed one of the 514 major drawbacks of using such methods to infer larger networks. From this aspect, JacLy is more 515 suitable as a small-scale $(<20$ metabolites) network inference method. There are several network 516 inference methods in the literature with a specific focus on small-scale networks (Weber et al., 517 2013; Linde et al., 2015). Since different cellular functions are biologically attached to smaller 518 metabolic pathways or subnetworks, it still makes sense to be able to infer active subnetworks 519 for a specific cellular condition rather than inferring the whole network. Table 2 summarizes 520 some characteristics of JacLy through a comparison with GGM as one of the most common 521 methods in inference of biological networks.

522

523 Currently, steady-state metabolome measurement data that are reported in the literature are 524 limited in terms of the number of replicates. This limitation is not specific to our method; 525 commonly used correlation-based inference methods are also suffering from low number of data 526 replicates and usually lead to significantly high number of false positives. Also, in case of real 527 metabolome data, experimental measurement errors interfere with natural stochasticity of the 528 system leading to lower quality in predicted networks. Moreover, since our method relies on the 529 fluctuation matrix (D) as one of its inputs, this external noise is more troublesome. To test the 530 applicability of our approach to the real metabolome data, we introduced random noise to the 531 SDE data of the yeast model by following the approach presented by Fuente et al. (2004). For 532 each metabolite, we sampled the random noise from a normal distribution with mean zero and a 533 standard deviation equal to $10 \%$ of its variance in the data. We generated 10 sets of noisy data 534 using this approach. The in silico data already includes randomness due to natural stochasticity 535 since it was generated using an SDE simulation toolbox. This random noise was still added to the 536 data to count for the other sources of error and randomness in the data, such as measurement 537 errors. We then applied both Jacly and GGM on the noisy data sets and compared the inference 538 results with that of noise-free SDE data. The average g-score dropped from 0.78 (noise-free data) 539 to 0.71 (noisy datasets) for JacLy and from 0.82 to 0.79 for GGM. These results provide a 540 theoretical base for applicability of our approach to real metabolome data that includes other 
541 sources of randomness in addition to the natural stochasticity of the system. Although the results

542 for GGM are better than those of JacLy, one should remember that JacLy clearly outperforms 543 GGM for lower number of data replicates (see Results section).

544

\section{Conclusions}

546 Thanks to the improvements introduced to the network inference algorithm, results reported in 547 the previous work (Öksüz, Sadıkoğlu \& Çakır, 2013) could be obtained much faster, with much 548 higher reproducibility, and with a higher prediction power. In addition, by applying the approach 549 to in silico metabolome data, we showed that the use of standard deviation of replicates is a 550 suitable approximation for the fluctuation matrix used as input to the algorithm. However, there 551 might be more sophisticated ways of estimating a fluctuation matrix that better represents the 552 nature of stochasticity in cellular metabolism. Finding more relevant fluctuation matrices for 553 different biological networks can be an altogether separate research topic and can lead to an 554 increase in accuracy and applicability of Lyapunov based inference methods such as JacLy. 555 Also, the power of JacLy was especially obvious when a considerably lower number of 556 replicates were used, or when a small portion of non-existent edges were introduced as prior 557 knowledge. Prediction of the Jacobian matrix from steady-state data is another power of JacLy 558 over GGM since Jacobian matrix is much more informative and biologically relevant in terms of 559 the network structure. Here, we have introduced JacLy as an algorithm to infer molecular 560 interactions of small networks since the size of matrix A is proportional to the square of the 561 network size, leading to dramatic increase in the computational time with respect to the network 562 size. Therefore, it should be applied with caution for metabolic systems having higher than 20 563 metabolites. Additionally, albeit its remarkably better performance for lower number or 564 replicates compared to a correlation-based inference as shown in this work, the use of JacLy for 565 datasets with lower than 100 replicates should be cautioned.

\section{References}

Bordbar A., Nagarajan H., Lewis NE., Latif H., Ebrahim A., Federowicz S., Schellenberger J., biomolecular interactions. Molecular Systems Biology 10:737. 
572 Çakır T., Hendriks MMWB., Westerhuis JA., Smilde AK. 2009. Metabolic network discovery 573 through reverse engineering of metabolome data. Metabolomics 5:318-329. DOI: 10.1007/s11306-009-0156-4.

575

Çakır T., Khatibipour MJ. 2014. Metabolic Network Discovery by Top-Down and Bottom-Up

576

577

578

579

580

581

582

583

584

585

586

587

588

589

590

591

592

593

594

595

596

597

598

599

600

601

602 Approaches and Paths for Reconciliation. Frontiers in Bioengineering and Biotechnology 2:62. DOI: 10.3389/fbioe.2014.00062.

Chassagnole C., Noisommit-Rizzi N., Schmid JW., Mauch K., Reuss M. 2002. Dynamic modeling of the central carbon metabolism of Escherichia coli. Biotechnology and Bioengineering 79:53-73.

de la Fuente A., Bing N., Hoeschele I., Mendes P. 2004. Discovery of meaningful associations in genomic data using partial correlation coefficients. Bioinformatics (Oxford, England) 20:3565-3574. DOI: 10.1093/bioinformatics/bth445.

Hendrickx DM., Hendriks MMWB., Eilers PHC., Smilde AK., Hoefsloot HCJ. 2011. Reverse engineering of metabolic networks, a critical assessment. Molecular bioSystems 7:511520. DOI: $10.1039 / \mathrm{c} 0 \mathrm{mb} 00083 \mathrm{c}$.

Hendriks MMWB., Eeuwijk FA van., Jellema RH., Westerhuis JA., Reijmers TH., Hoefsloot HCJ., Smilde AK. 2011. Data-processing strategies for metabolomics studies. TrAC Trends in Analytical Chemistry 30:1685-1698. DOI: 10.1016/j.trac.2011.04.019.

Jamshidi N., Palsson B. 2008. Formulating genome-scale kinetic models in the post-genome era. Molecular Systems Biology 4:171.

Kampen NGV. 1992. Stochastic Processes in Physics and Chemistry. Elsevier.

Kresnowati MTAP., van Winden WA., Almering MJH., ten Pierick A., Ras C., Knijnenburg TA., Daran-Lapujade P., Pronk JT., Heijnen JJ., Daran JM. 2006. When transcriptome meets metabolome: fast cellular responses of yeast to sudden relief of glucose limitation. Molecular Systems Biology 2:49. DOI: 10.1038/msb4100083.

Kügler P., Yang W. 2014. Identification of alterations in the Jacobian of biochemical reaction networks from steady state covariance data at two conditions. Journal of Mathematical Biology 68:1757-1783. DOI: 10.1007/s00285-013-0685-3.

Linde J., Schulze S., Henkel SG., Guthke R. 2015. Data- and knowledge-based modeling of gene regulatory networks: an update. EXCLI Journal 14:346-378. DOI: 10.17179/excli2015168. 
603 Mangan NM., Brunton SL., Proctor JL., Kutz JN. 2016. Inferring Biological Networks by Sparse 604 Identification of Nonlinear Dynamics. IEEE Transactions on Molecular, Biological and 605 Multi-Scale Communications 2:52-63. DOI: 10.1109/TMBMC.2016.2633265.

606 Montastier E., Villa-Vialaneix N., Caspar-Bauguil S., Hlavaty P., Tvrzicka E., Gonzalez I., Saris 607 WHM., Langin D., Kunesova M., Viguerie N. 2015. System Model Network for Adipose 608 Tissue Signatures Related to Weight Changes in Response to Calorie Restriction and 609 10.1371/journal.pcbi.1004047.

Öksüz M., Sadıkoğlu H., Çakır T. 2013. Sparsity as cellular objective to infer directed metabolic networks from steady-state metabolome data: a theoretical analysis. PloS One 8:e84505. DOI: 10.1371/journal.pone.0084505.

Picchini U. 2007. SDE Toolbox: Simulation and Estimation of Stochastic Differential Equations with MATLAB.

Scott M. 2013. Applied stochastic processes in science and engineering.

Shao B., Wu J., Tian B., Ouyang Q. 2015. Minimum network constraint on reverse engineering to develop biological regulatory networks. Journal of Theoretical Biology 380:9-15. DOI: $10.1016 /$ j.jtbi.2015.05.005.

Srividhya J., Crampin EJ., McSharry PE., Schnell S. 2007. Reconstructing biochemical pathways from time course data. Proteomics 7:828-838. DOI: 10.1002/pmic.200600428.

Steuer R., Kurths J., Fiehn O., Weckwerth W. 2003. Observing and interpreting correlations in metabolomic networks. Bioinformatics (Oxford, England) 19:1019-1026.

Sun X., Länger B., Weckwerth W. 2015. Challenges of Inversely Estimating Jacobian from Metabolomics Data. Frontiers in Bioengineering and Biotechnology 3:188. DOI: 10.3389/fbioe.2015.00188.

Sun X., Weckwerth W. 2012. COVAIN: a toolbox for uni- and multivariate statistics, time-series and correlation network analysis and inverse estimation of the differential Jacobian from metabolomics covariance data. Metabolomics 8:81-93. DOI: 10.1007/s11306-012-03993.

Tegnér J., Björkegren J. 2007. Perturbations to uncover gene networks. Trends in genetics: TIG 23:34-41. DOI: 10.1016/j.tig.2006.11.003. 
633 Teusink B., Passarge J., Reijenga CA., Esgalhado E., van der Weijden CC., Schepper M., Walsh

634

635

636

637

638

639

640

641

642

643

644

645

646

647

648

649

650

651
MC., Bakker BM., van Dam K., Westerhoff HV., Snoep JL. 2000. Can yeast glycolysis be understood in terms of in vitro kinetics of the constituent enzymes? Testing biochemistry. European Journal of Biochemistry 267:5313-5329.

Wang T., Ren Z., Ding Y., Fang Z., Sun Z., MacDonald ML., Sweet RA., Wang J., Chen W. 2016. FastGGM: An Efficient Algorithm for the Inference of Gaussian Graphical Model in Biological Networks. PLoS computational biology 12:e1004755. DOI: 10.1371/journal.pcbi. 1004755 .

Weber M., Henkel SG., Vlaic S., Guthke R., van Zoelen EJ., Driesch D. 2013. Inference of dynamical gene-regulatory networks based on time-resolved multi-stimuli multiexperiment data applying NetGenerator V2.0. BMC systems biology 7:1. DOI: 10.1186/1752-0509-7-1.

Wu L., Mashego MR., van Dam JC., Proell AM., Vinke JL., Ras C., van Winden WA., van Gulik WM., Heijnen JJ. 2005. Quantitative analysis of the microbial metabolome by isotope dilution mass spectrometry using uniformly 13C-labeled cell extracts as internal standards. Analytical Biochemistry 336:164-171. DOI: 10.1016/j.ab.2004.09.001.

Yeung MKS., Tegnér J., Collins JJ. 2002. Reverse engineering gene networks using singular value decomposition and robust regression. Proceedings of the National Academy of Sciences of the United States of America 99:6163-6168. DOI: 10.1073/pnas.092576199. 


\section{Table 1 (on next page)}

Inference results for the in-silico data, comparison of JacLy and GGM 
Table 1: Inference results for the in-silico data, comparison of JacLy and GGM

\begin{tabular}{|c|c|c|c|c|c|c|}
\hline & \multicolumn{3}{|c|}{ In-silico data for Yeast } & \multicolumn{3}{c|}{ In-silico data for E.coli } \\
\hline & TPR & FPR & g-score & TPR & FPR & g-score \\
\hline JacLy & 0.66 & 0.08 & 0.78 & 0.69 & 0.29 & 0.70 \\
\hline GGM & 0.76 & 0.12 & 0.82 & 0.63 & 0.12 & 0.74 \\
\hline
\end{tabular}

2

3 
Table 2 (on next page)

A summarized comparison of JacLy with GGM 


\begin{tabular}{|c|c|c|}
\hline & JacLy & $G G M$ \\
\hline $\begin{array}{l}\text { Computational time versus } \\
\text { network size }\end{array}$ & $\begin{array}{ll}- & \text { NP-hard problem } \\
- & \text { Computational time } \\
\text { increases exponentially by } \\
\text { increasing the network } \\
\text { size }\end{array}$ & $\begin{array}{l}\text { No sensible change in the } \\
\text { computational time from very } \\
\text { small to very large networks }\end{array}$ \\
\hline $\begin{array}{l}\text { Accuracy versus number of data } \\
\text { replicates }\end{array}$ & $\begin{array}{l}\text { - Accuracy is a moderate } \\
\text { function of number of } \\
\text { data replicates } \\
\text { - } \quad \text { For lower number of data } \\
\text { replicates, it outperforms } \\
\text { correlation based methods }\end{array}$ & $\begin{array}{l}\text { - } \\
\text { Accuracy is a very } \\
\text { strong function of } \\
\text { number of data } \\
\text { replicates } \\
\text { - } \\
\text { Reduction in the } \\
\text { number of data } \\
\text { replicates has a very } \\
\text { high negative impact } \\
\text { on the quality of } \\
\text { inferred network }\end{array}$ \\
\hline Directionality of inferred network & Directed & Undirected \\
\hline $\begin{array}{l}\text { Meaningfulness of inferred edge's } \\
\text { weights }\end{array}$ & $\begin{array}{l}\text { - Mechanistically } \\
\text { meaningful } \\
\text { - } \quad \text { Inferred values for the } \\
\text { Jacobian elements are } \\
\text { measures of interaction } \\
\text { strengths and their sign } \\
\text { (positive/negative) } \\
\text { points into the nature of } \\
\text { interaction }\end{array}$ & $\begin{array}{l}\text { - Correlation values } \\
\text { cannot be used as any } \\
\text { physical or } \\
\text { mechanistical } \\
\text { parameter of the } \\
\text { system }\end{array}$ \\
\hline
\end{tabular}

4 


\section{Figure 1}

Use of a community of the best-found Jacobians to infer the structure of the network.

A) selecting a bounded area around the elite Jacobian based on a percentage of the number of zeros in the elite Jacobian and the residual of equation 4. B) a schematic example of alignment and combination of Jacobian vectors in the selected community to come up with the final structure.

A

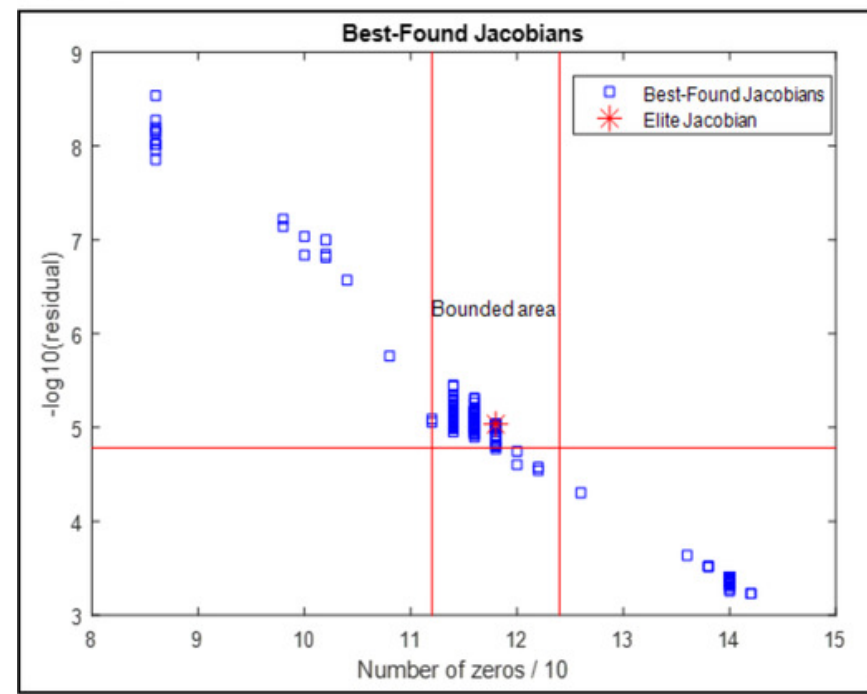

B

\begin{tabular}{|c|c|c|c|c|c|c|c|c|c|c|c|c|}
\hline \multirow{10}{*}{ 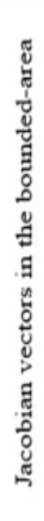 } & Jacobian vector 1 & 1 & 1 & 0 & 1 & 0 & 0 & $\ldots$ & 1 & 0 & 1 & 0 \\
\hline & Jacobian vector 2 & 0 & 1 & 0 & 1 & 1 & 0 & $\cdots$ & 0 & 0 & 1 & 0 \\
\hline & Jacobian vector 3 & 1 & 1 & 1 & 0 & 0 & 0 & $\cdots$ & 0 & 0 & 0 & 1 \\
\hline & Jacobian vector 4 & 1 & 1 & 0 & 0 & 1 & 1 & $\cdots$ & 1 & 0 & 0 & 0 \\
\hline & Jacobian vector 5 & 0 & 1 & 0 & 0 & 1 & 0 & $\cdots$ & 1 & 0 & 1 & 1 \\
\hline & Jacobian vector 6 (elite) & 1 & 1 & 1 & 0 & 0 & 0 & $\cdots$ & 1 & 0 & 0 & 1 \\
\hline & Jacobian vector 7 & 0 & 1 & 0 & 0 & 1 & 1 & $\cdots$ & 0 & 0 & 0 & 1 \\
\hline & Jacobian vector 8 & 0 & 1 & 1 & 1 & 0 & 0 & $\cdots$ & 1 & 0 & 1 & 1 \\
\hline & Jacobian vector 9 & 1 & 1 & 0 & 0 & 0 & 1 & $\cdots$ & 1 & 0 & 1 & 0 \\
\hline & Jacobian vector 10 & 1 & 1 & 0 & 0 & 1 & 0 & $\cdots$ & 0 & 0 & 0 & 1 \\
\hline & & 0.6 & 1 & 0.3 & 0.3 & 0.5 & 0.3 & $\ldots$ & 0.6 & 0 & 0.5 & 0.6 \\
\hline & ture of the final Jacobian & 1 & 1 & 0 & 0 & 1 & 0 & $\ldots$ & 1 & 0 & 1 & 1 \\
\hline
\end{tabular}

